\title{
Protective effects of folic acid against central nervous system neurotoxicity induced by lead exposure in rat pups
}

\author{
F.S. Quan, X.F. Yu, Y. Gao and W.Z. Ren \\ College of Animal Sciences, Jilin University, Changchun, China \\ Corresponding author: W. Ren \\ E-mail: renwenzhi_rwz@163.com \\ Genet. Mol. Res. 14 (4): 12466-12471 (2015) \\ Received February 23, 2015 \\ Accepted July 2, 2015 \\ Published October 16, 2015 \\ DOI http://dx.doi.org/10.4238/2015.October.16.13
}

\begin{abstract}
Recent studies found folic acid is associated with lower blood lead $(\mathrm{Pb})$ levels, and folate deficient children are more susceptible to the negative cognitive effects of $\mathrm{Pb}$. This study evaluated the protective effects of folate supplementation against $\mathrm{Pb}$ exposure in rat pups and the mechanisms of protection. A total of 72 rats were used. Thirty were administered $\mathrm{Pb}$ only; 30, $\mathrm{Pb}$ and folic acid at the same time; and 12, only physiological saline. Protective effects of folic acid were examined at 14 , 21 , and 28 days after treatment. Lower blood $\mathrm{Pb}$ levels were found in all of the samples collected from the rats treated with folic acid. Downregulation of Bc1-2 expression and upregulation of Bax expression were observed in the neurons of folic acid-treated rats. Significantly more hematoxylin and eosin stained neurons were found in the folic acid treatment group. Nuclear enrichment and neuron apoptosis were observed by electron microscopy in the Pb-treated group. In conclusion, this study demonstrated that folic acid supplementation might offer efficient protective effects against $\mathrm{Pb}$ poisoning in rat pups, which was associated with less neuron damage and lower blood levels of $\mathrm{Pb}$.
\end{abstract}

Key words: Rat pups; Lead poisoning; Central nervous system; Folic acid 


\section{INTRODUCTION}

Lead $(\mathrm{Pb})$ is a kind of affinity poison. Increased levels of $\mathrm{Pb}$ in the body can cause serious damage in humans and other vertebrates. $\mathrm{Pb}$ is toxic to many organs and tissues, including the nervous system. $\mathrm{Pb}$ is particularly toxic to children, causing potentially permanent learning and behavior disorders. $\mathrm{Pb}$ poisoning is a long-term process. The half-life of $\mathrm{Pb}$ is about 10 years and it is difficult to excrete from the body, which can result in body $\mathrm{Pb}$ levels being five times higher than in the environment. Although the ideal level of $\mathrm{Pb}$ in the body is zero, most people have some $\mathrm{Pb}$ derived from the environment (Ahamed and Siddiqui, 2007). In children, the blood-brain barrier is not complete, the central nervous system is relatively fragile, and excretion from the central nervous system is not perfect. All of this makes the child central nervous system highly vulnerable to $\mathrm{Pb}$ damage (Bennet et al., 2007; Bokara et al., 2009). If a large amount of $\mathrm{Pb}$ is taken by a child, it can cause pathological damage to the brain tissues, such as cell edema, hemorrhage, demyelination, degeneration, and hippocampal formation atrophy, possibly leading to intellectual impairment. Therefore, $\mathrm{Pb}$ poisoning has always drawn the attention of the public (Massó-González and Antonio-García, 2009). Presently, chelating agents are used as the main treatment in $\mathrm{Pb}$ poisoning, but these agents have serious side effects, including renal damage (Garza et al., 2006; Ahamed and Siddiqui, 2007; Bennet et al., 2007; Bokara et al., 2009; MassóGonzález and Antonio-García, 2009).

Several reports have linked high blood $\mathrm{Pb}$ levels with folic acid deficiency (Rader et al., 1982; Lee et al., 2005; Solon et al., 2008). More importantly, folate deficient children are more susceptible to intellectual impairment by $\mathrm{Pb}$ (Lee et al., 2005). These studies suggest folate supplementation might offer some protective effects against $\mathrm{Pb}$ exposure. However, there are still no experimental studies that test this hypothesis, especially in young intelligence-developing animal models. In this study, rat pups were used to study the protective effects of folic acid on central nervous system neurotoxicity induced by $\mathrm{Pb}$ exposure.

\section{MATERIAL AND METHODS}

\section{Animals and treatments}

Three-week-old Sprague-Dawley rats weighing 45-60 g were bought from the experimental animal center of the Medical College of Jilin University. The rats were randomly divided into three groups and maintained at constant temperature $\left(22^{\circ} \mathrm{C}\right)$ and humidity $(60 \%)$. One group (30 rats) received $0.1 \% \mathrm{~Pb}$ acetate in their drinking water (Group I). The second group (30 rats) was administrated $0.4 \mathrm{mg} / \mathrm{kg}$ of folic acid orally once a day in addition to receiving the same concentration of $\mathrm{Pb}$ acetate in their drinking water (Group II). The last group (12 rats) received sterile physiological saline as control (Group III). Ten rats from Groups I and II and four rats from Group III were killed to examine the protective effects of folic acid at 14, 21, and 28 days after treatment.

\section{Behavioral testing}

Rats were evaluated using the Y-maze test, as described in a previous report. Briefly, one rat was tested at a time. Three lamps at the arms were turned on to let the rat adapt to new environment for $1 \mathrm{~min}$ before the lights were turned off. The test was performed by randomly turning on the light in one of the arms, which housed no rat, for $5 \mathrm{~s}$ (the arm with the light on 
is called the "safe zone"), then the other two arms were galvanized with electric current (50 V). Escape time to the safe zone was recorded for each rat ( $<30 \mathrm{~s}$ was regarded as success, otherwise as failure). Finally, the lights were turned on for $30 \mathrm{~s}$ and then turned off. After a 1-min interval, the test was repeated until the rat learned to escape to the safety zone successfully ( 9 of 10 times). The number of repeat tests for successful learning was recorded. All rats underwent $Y$ maze testing 3 days before they were killed. Rats were trained with one round of the $Y$ maze everyday.

\section{Blood $\mathrm{Pb}$ level measurement}

Cardiac blood was collected for measurement of $\mathrm{Pb}$ levels, which was measured using graphite furnace atom absorption spectrometry (Lee et al., 2005).

\section{Histopathologic evaluation}

Half of the rats in each group were anesthetized by ip injection of $4.0 \%$ chloral hydrate and perfused with $4 \%$ paraformaldehyde in $0.1 \mathrm{M}$ phosphate buffer. Brains were removed and embedded in paraffin blocks. Paraffin sections $(5 \mu \mathrm{m})$ were prepared and stained with hematoxylin and eosin. Histological changes in the hippocampus and cerebellum were observed under a microscope.

\section{Western blot analysis}

Half of the rats in each group were used for western blot analysis. The hippocampus and cerebellum were separated on ice. After washing with $0.9 \%$ sodium chloride, the clean hippocampus and cerebellum were preserved in liquid nitrogen until use. A protein preparation kit was used to exact the protein, and the concentration was measured by the Bradford method. Twenty micrograms of each protein were subjected to SDS-PAGE for $2 \mathrm{~h}$. The gel was then electrically transferred to a PVDF membrane. After blocking for $1 \mathrm{~h}$, the PVDF membrane was incubated with $\alpha-\mathrm{Bcl}-2$, $\alpha-B a x$, and $\alpha-\beta$-actin antibodies at $4^{\circ} \mathrm{C}$ overnight. PVDF membranes were then incubated with goat anti-rat HRP secondary antibody for $1 \mathrm{~h}$. After washing three times, ECL chemiluminescent agent was used as a substrate and exposed to an X-ray film. The signal was quantified using the Sensi Ansis software.

\section{Data processing}

Data were processed statistically using the SPSS software.

\section{RESULTS}

\section{Effects of folic acid on body weight}

The body weights of rats in Group II decreased significantly compared to the other two groups, as analyzed by a Student $t$-test. No feature differences were observed in all groups (Table 1). 


\begin{tabular}{|c|c|c|c|c|}
\hline \multirow[t]{2}{*}{ Group } & \multirow[t]{2}{*}{ Number of Animals } & \multicolumn{3}{|c|}{ Body weight (g) } \\
\hline & & 1 week & 2 weeks & 3 weeks \\
\hline Group I & 30 & $19.6 \pm 2.1$ & $32.1 \pm 2.1$ & $49.1 \pm 5.9$ \\
\hline Group II & 30 & $17.7 \pm 2.7$ & $30.1 \pm 3.1$ & $47.9 \pm 3.4^{*}$ \\
\hline Group III & 12 & $21.7 \pm 0.8$ & $34.9 \pm 3.2$ & $52.8 \pm 6.6$ \\
\hline
\end{tabular}

Values are reported as means \pm SEM of rats in each group. * $\mathrm{P}<0.05$ versus Group I and III.

\section{Effects of folic acid on Y-maze performance}

Significant differences were observed between the Pb-treated, folic acid-treated, and control groups $(\mathrm{P}<0.05$, Table 2$)$. The number of repeat trainings needed decreased in the $\mathrm{Pb}$ treated group, folic acid treatment group, and control group.

Table 2. Repeat number for Y-maze training.
\begin{tabular}{lcccc}
\hline Group & Number of Animals used & 1 week & 2 weeks & 3 weeks \\
\hline Group I & 12 & $49.14 \pm 2.87$ & $45.16 \pm 1.64$ & $44.18 \pm 1.45$ \\
Group II & 30 & $55.62 \pm 1.02$ & $71.23 \pm 2.03^{*}$ & $92.54 \pm 0.92^{*}$ \\
Group III & 30 & $54.26 \pm 1.81$ & $64.32 \pm 1.13^{\star \Delta}$ & $72.64 \pm 1.75^{* \Delta}$ \\
\hline
\end{tabular}

Student $t$ - test compared to Group I, ${ }^{*} \mathrm{P}<0.05$; compared to Group II, ${ }^{\Delta} \mathrm{P}<0.05$.

\section{Effect of folic acid on blood $\mathrm{Pb}$ levels}

Compared to the physiological saline group, significantly higher blood $\mathrm{Pb}$ levels were observed in the Pb-treated group in weeks 1,2 , and 3 after the experiment $(P<0.05)$. Significantly lower $\mathrm{Pb}$ levels were observed in the folic acid-treated group than the $\mathrm{Pb}$-treated group, with longer treatment showing lower blood $\mathrm{Pb}$ levels (Table 3).

\begin{tabular}{|c|c|c|c|c|}
\hline Grouping & Number of Animals used & 1 week & 2 weeks & 3 weeks \\
\hline Group I & 4 & $0.19 \pm 0.04$ & $0.27 \pm 0.02$ & $0.23 \pm 0.05$ \\
\hline Group II & 10 & $4.26 \pm 0.02^{\star}$ & $4.59 \pm 0.05^{\star}$ & $4.96 \pm 0.03^{*}$ \\
\hline Group III & 10 & $3.71 \pm 0.01^{* \Delta}$ & $3.62 \pm 0.02^{* \Delta}$ & $3.48 \pm 0.03^{* \Delta}$ \\
\hline
\end{tabular}

Student $t$-test compared to Group I, *P $<0.05$; compared to Group II, ${ }^{\Delta} \mathrm{P}<0.05$.

\section{Effects of folic acid on $\mathrm{Pb}$-induced neuronal damage}

As seen in Figure 1, the control group showed a bigger nucleus-to-cytoplasm ratio in the hippocampal neurons; the cell nuclei were round or oval and stained evenly with light blue-purple color, and the nucleoli were stained darker. In the CA1 zone, the pyramidal cells were in 3-4 layers. Compared to the control group, the number of dentate gyrus neurons in the hippocampal CA 1-3 zones decreased significantly in the Pb-treated group, often accompanied with shrinking of the cell body, condensed cytoplasm, karyopyknosis, and whole-cell staining deep red, which was most notable in the CA1 zone, CA3 zone, and dentate gyrus. More neurons and fewer cells with strong cytoplasmic staining were observed in the folic acid-treated animal samples than in the Pb-treated group. In the Pb-treated group, more damaged Purkinje cells with condensed nuclei were observed in the cerebellum. 


\section{Effects of folic acid on the expression of $\mathrm{Bcl}-2$ and Bax}

As demonstrated in Figure 2, higher $\mathrm{Bcl}-2$ protein expression was observed in the $\mathrm{Pb}$ treated group compared to control by quantitative western blot. However, Bcl-2 expression was lower than in the folic acid treatment group. Higher Bax protein expression was observed in both $\mathrm{Pb}$-treated and folic acid-treated groups compared to the control group, and Bax was higher in the $\mathrm{Pb}$-treated group compared to the folic acid-treated group (Figure 3A and 3B).

\section{DISCUSSION}

The hippocampus plays a key part in learning and the cerebellum is an important regulation center for movement. Both are targeted organs for Pb poisoning (Han et al., 2007; Yin et al., 2008). In our experiment, compared to folic acid-treated animals, more denatured neurons in the hippocampus were observed in Pb-treated rats. Additionally, the longer folic acid was given, the less the damage done. Results in the cerebellum were similar to those of the hippocampus. Under electron microscopy observation, folic acid can remit and suppress the continuation of damage done to neurons. Compared to the control group, Pb-treated rats demonstrated unresponsiveness, slowness, as well as less and slower weight gains. Signs of improvement were observed in the folic acid-treated group. Folic acid showed a certain degree of anti-cell damage effects, suggesting it could be used to protect neurons from $\mathrm{Pb}$ poisoning (in experimental animals).

The Y-maze test is often used to test an animal's IQ. This behavior test is used to test rodent spatial recognition and memory functions. The advantages of the $Y$ maze test over the passive avoidance test are as follows: 1 ) it makes use of a rodent's instinct to explore new environments, and 2) the tested animals do not need to learn any rules to escape the threat, thus it can effectively reflect the animal's recognition and memory capabilities to adapt to new environments (Dellu et al., 2000). In our experiment, all folic acid-treated animals needed fewer repeats to reach the safety zone than $\mathrm{Pb}$-treated animals, even though animals in both groups needed significantly more repeats to reach the safety zone than the control group $(P<0.05)$. The results demonstrate that to a certain degree, folic acid could help $\mathrm{Pb}$ poisoned rats recover from IQ damage.

The active form of folic acid in the body is tetrahydrofolate, which is used in one-carbon unit transfer reactions, in the synthesis of purines and pyrimidines, and in mutual conversion between amino acids. Thus, folic acid has important regulative effects on ganglion neurogenesis and NSC proliferation and differentiation (Hsu and Guo 2002; Soltaninejad et al., 2003). Results demonstrated that in the $\mathrm{Pb}$-treated group, apoptosis stimulated a continuous increase of Bax protein and suppressed $\mathrm{Bcl}-2$ expression. Because folic acid can bind to $\mathrm{Pb}$, resulting in increased excretion from the body, and because of folic acid's anti-oxidative effect, the effects of Pb poisoning were suppressed by folic acid treatment. It was also observed that the longer the animals were treated with folic acid, the more Bcl-2 was expressed, the more apoptosis was suppressed, and the weaker Bax was expressed. All these demonstrate that folic acid has protective effects on brain neurons from $\mathrm{Pb}$ poisoning. The mechanism of folic acid's anti-apoptotic effects maybe associated with suppression of Bax gene expression, which subsequently stimulates Bcl-2 expression.

The pathological lesions in Pb-poisoned patients are largely caused by increased oxidative stress. Several studies have documented that antioxidants can protect patients against $\mathrm{Pb}$ poisoning (Yin et al., 2008). The antioxidant activity of folic acid, which is effective in eliminating hydroxyl free radicals and superoxide anions, has been demonstrated previously. As a member of the vitamin B family, folic acid is also an important neurotrophic factor that contributes greatly to 
the development of neurons and brain cells in infants, leading to increased intellectual capability. Folic acid is also reported to improve $\mathrm{Pb}$ excretion and may make it harder for $\mathrm{Pb}$ to bind to blood elements (Solon et al., 2008).

This experiment demonstrated that because folic acid increases the synthesis of $\mathrm{NO}$ and has anti-oxidative effects; taking folic acid orally could have significant treatment effects on $\mathrm{Pb}$ poisoning. Folic acid may increase the synthesis of $\mathrm{NO}$ in the body, leading to increased serum NO activity and increased anti-oxidative effects in the organism. This subsequently renders protective effects by preventing neuron apoptosis in $\mathrm{Pb}$ poisoned rats. As further clinical research unfolds, folic acid, an effective, cheap, and safe drug can be used for early prevention and treatment of $\mathrm{Pb}$ poisoning. However, the long-term side effects of folic acid use are yet to be determined.

\section{Conflicts of Interest}

The authors declare no conflict of interest.

\section{ACKNOWLEDGEMENTS}

Research supported by the "985" Project of Jilin University and the Youth Research Fund project in Jilin Province.

\section{REFERENCES}

Ahamed M and Siddiqui MKJ (2007). Low level lead exposure and oxidative stress current opinions. Clin. Chim. Acta 383: 57-64. Bennet C, Bettaiya R, Rajanna S, Baker L, et al. (2007). Region specific increase in the antioxidant enzymes and lipid peroxidation products in the brain of rats exposed to lead. Free Radic. Res 41: 267-273.

Bokara KK, Blaylock I, Denise SB, Bettaiya R, et al. (2009). Influence of lead acetate on glutathione and its related enzymes in different regions of rat brain. J. Appl. Toxicol. 29: 452-458.

Dellu F, Contarino A, Simon H, Koob GF, et al. (2000). Genetic differences in response to novelty and spatial memory using a two-trial recognition task in mice. Neurobiol. Learn. Mem. 73: 31-48.

Garza A, Vega R and Soto E (2006). Cellular mechanisms of lead neurotoxicity. Med. Sci. Monit.12: 57-65.

Han JM, Chang BJ, Li TZ, Choe NH, et al. (2007). Protective effects of ascorbic acid against lead-induced apoptotic neurodegeneration in the developing rat hippocampus in vivo. Brain. Res. 185: 68-74.

Hsu PC and Guo YL (2002). Antioxidant nutrients and lead toxicity. Toxicology 180: 33-44.

Lee MG, Chun OK and Song WO (2005). Determinants of the Blood Lead Level of US Women of Reproductive Age. J. Am. Coll. Nutr. 24: 1-9.

Massó-González EL and Antonio-García MT (2009). Natural antioxidants protect against lead-induced damage during pregnancy and lactation in rat's pups. Ecotoxicol. Environ. Saf. 72: 2137-2142.

Rader JI, Celesk EM, Peeler JT and Mahaffey KR (1982). Effect of lead acetate on rats fed diets containing low levels of folic acid. Drug Nutr. Interact. 1: 131-142.

Solon O, Riddell TJ, Quimbo SA, Butrick E, et al. (2008). Associations between Cognitive Function, Blood Lead Concentration, and Nutrition among Children in the Central Philippines. J. Pediatr. 152: 237-243.

Soltaninejad K, Kebriaeezadeh A, Minaiee B, Ostad SN, et al. (2003). Biochemical and ultrastructural evidences for toxicity of lead through free radicals in rat brain. Hum. Exp. Toxicol. 22:417-423.

Yin ST, Tang ML, Su L, Chen L, et al. (2008). Effects of epigallocatechin-3-gallate on lead-induced oxidative damage. Toxicology 249: 45-54. 